\title{
Effect of Particle Size on the Wear Property of Magnetorheological Fluid
}

\author{
Qiuxiang Zhang, ${ }^{1}$ Xinhua Liu, ${ }^{1,2}$ Yankun Ren, ${ }^{1}$ Lifeng Wang, ${ }^{1}$ and Yuan Hu${ }^{1}$ \\ ${ }^{1}$ School of Mechanical and Electrical Engineering, China University of Mining \& Technology, Xuzhou 221116, China \\ ${ }^{2}$ The State Key Laboratory of Tribology, Tsinghua University, Beijing 100000, China \\ Correspondence should be addressed to Xinhua Liu; 1_xinhua_2006@126.com
}

Received 12 February 2016; Revised 3 May 2016; Accepted 9 May 2016

Academic Editor: Paolo Ferro

Copyright (c) 2016 Qiuxiang Zhang et al. This is an open access article distributed under the Creative Commons Attribution License, which permits unrestricted use, distribution, and reproduction in any medium, provided the original work is properly cited.

\begin{abstract}
Aiming to study the effect of particle size on the wear property of magnetorheological fluid (MRF), experiment materials, preparation process, and test methods are elaborated, and three different MRF samples consisting of particles of different size are prepared. Test experiments are carried out and the effect of particle size on the wear property of MRF is discussed. Moreover, the microstructures of particles extracted from MRF obtained before and after the wear experiments are observed by scanning electron microscope (SEM). Experimental results show that the particle size has a significant effect on wear property of MRF. Furthermore, the MRF with particles of 1.5-2.8 $\mu \mathrm{m}$ diameter on average is good for the requirement of engineering applications.
\end{abstract}

\section{Introduction}

Magnetorheological fluid (MRF) is a new type of intelligent materials, which is a suspension consisting of carrier liquid, soft magnetic particles, and surfactant [1-4]. Without additional magnetic field, MRF appears as Newtonian fluid with low viscosity and good fluidity. But its rheological properties will change significantly in the presence of external magnetic field: it will become solid-like, and these changes are instantaneous, controllable, and reversible [5-8]. Due to its unique characteristics, MRF has wide application in shock absorbers, transmission devices, brake apparatus, and medical equipment as well as aerospace materials [9-14]. But, according to relevant research, the torque capacity that transmission devices can transmit will decline [15-17], and sedimentation stability as well as zero-field viscosity of MRF will change after use. These changes are mainly caused by the wear of MRF which includes evaporation of liquid, destruction of the additive, and the wear of particles [4]. The small role of the first two factors makes the wear of particles crucial in affecting the properties of MRF. In particular, the wear of particles will decrease the shear stress of MRF greatly and be harmful to the stability and application of MRF.

In this paper, we expect to explore the effect of particle size on the wear property of MRF. The wear property covers the change of shear stress, sedimentation stability, and zerofield viscosity of MRF after use. As part of this study, a magnetorheological fluid transmission test-bed which aimed to conduct the wear experiments was designed and assembled in the laboratory. The shear stress of the MRF was investigated, and the sedimentation stability and zero-field viscosity were researched. In addition, the surface topography of magnetic particles was observed by SEM to interpret the possible wear mechanism of MRF.

The rest of this paper was organized as follows: Section 2 described the experimental methods and preparation of the MRF. Section 3 presented the results and discussion based on the wear experiments. Section 4 summarized the conclusions and future work.

\section{Experimental}

2.1. Experimental Methods for the Wear Property of MRF. The magnetorheological fluid transmission test-bed is shown in Figure 1. This test-bed mainly consists of a motor, two torque sensors, a magnetorheological fluid transmission device (MRFTD) designed by ourselves, a magnetic powder brake (MPB), a frequency converter, and a computer. The motor is the power source providing a settled speed for the input shaft. Upon performing the experiments, let the 


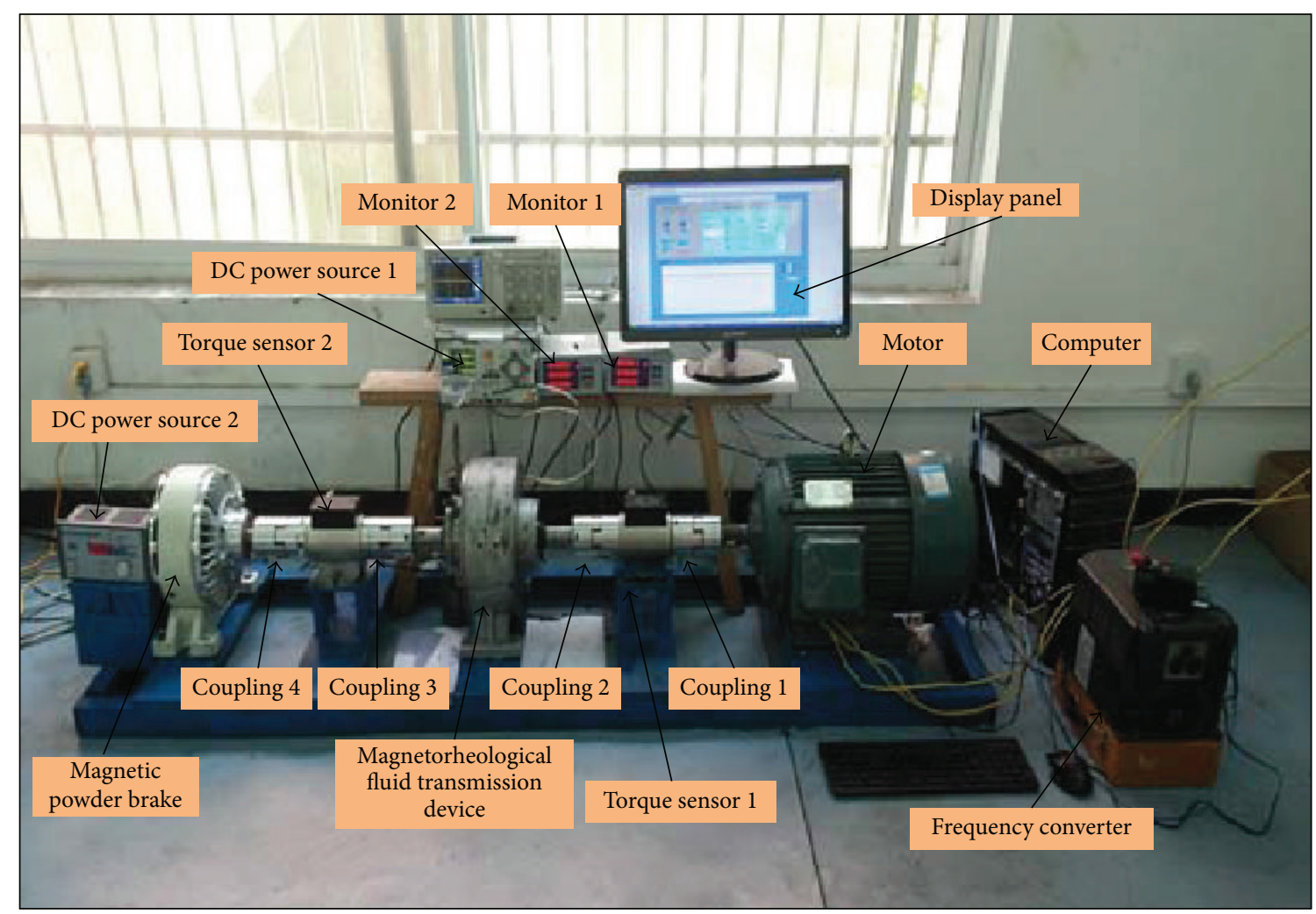

Figure 1: MRF test-bed.

TABLE 1: Three MRF samples' index and the corresponding parameter of particles.

\begin{tabular}{|c|c|c|c|c|c|}
\hline \multirow{2}{*}{ Index } & \multirow{2}{*}{ Particle model } & \multirow{2}{*}{$D_{\text {av }}$ (average diameter $) / \mu \mathrm{m}$} & \multicolumn{3}{|c|}{ Particle size distribution } \\
\hline & & & $D(0.1)$ & $D(0.5)$ & $D(0.9)$ \\
\hline MRF-15 & MPS-MRF-15 & $3-5$ & $\leq 2.5$ & $\leq 4.5$ & $<8$ \\
\hline MRF-25 & MPS-MRF-25 & $2.5-3.5$ & $\leq 2.0$ & $\leq 4.0$ & $<7$ \\
\hline MRF-35 & MPS-MRF-35 & $1.5-2.8$ & $\leq 1.5$ & $\leq 2.5$ & $<5$ \\
\hline
\end{tabular}

MRFTD work under the effect of constant magnetic field by regulating the exciting current at $3.5 \mathrm{~A}$. The exciting current is produced by adjustable DC power source. And the load of this system is set by adjusting the exciting current of MPB to $0.4 \mathrm{~A}$. Besides that, the torque sensors are used to get the torque and the rotative speed difference of working disks, and the data is collected by the computer. All experiments are carried out at room temperature.

2.2. Preparation of $M R F$. The preparation of MRF can be realized by existing technology $[18,19]$. The detailed steps can be described as follows. Firstly, carrier liquid and surfactant are mixed with certain proportion in a container. The mixture is stirred evenly under water-bathing at $50^{\circ} \mathrm{C}$ in a blender for 2 hours. Then, soft magnetic particles are poured slowly into the container while the blender is running. The particles are evenly distributed in the compound liquid after this step. Secondly, the mixture is milled in a planetary ball mill for about $10 \mathrm{~h}$. The aim of this step is to ensure that soft magnetic particles are coated sufficiently by surfactant. The whole process can be shown in Figure 2.

Keep the ingredients of MRF samples identical except for particle size. And the volume fractions of soft magnetic particles are $20 \%$. The details of soft magnetic particles (carbonyl iron powder, purchased from Jiangsu Tianyi Ultrafine Metal Powder co., Ltd.) and the index of samples are given in Table 1.

\section{Results and Discussion}

3.1. Shear Stress of MRF. The relation between shear stress $(\gamma)$ and transmitted torque of $\operatorname{MRF}(T)$ can be expressed as follows [20, 21]:

$$
T=\frac{2}{3} \pi \tau\left(r_{2}^{3}-r_{1}^{3}\right)+\frac{\pi \eta \Delta \omega}{2 h}\left(r_{2}^{4}-r_{1}^{4}\right)
$$

Here, $r_{1}$ is the inner radius of actuator disk, $r_{2}$ is the maximal radius of actuator disk, $\eta$ is the viscosity of carrier liquid in zero magnetic fields, $\Delta \omega$ is the rotative speed difference of 


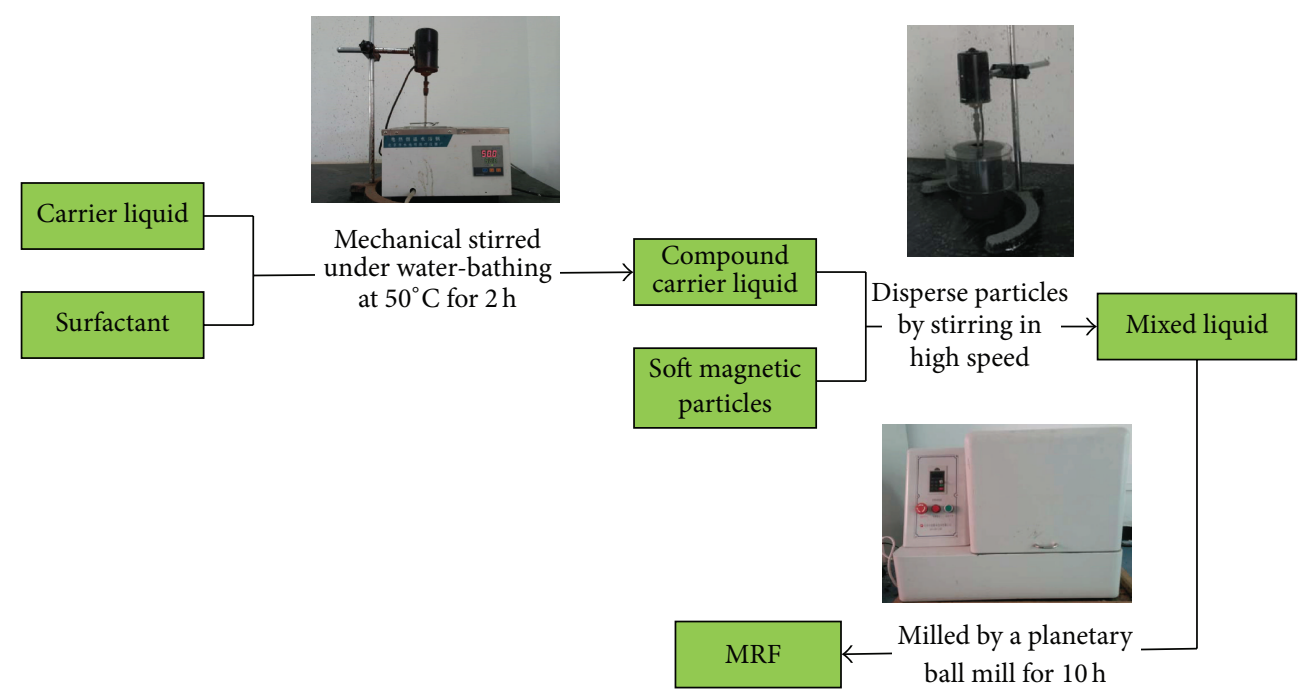

FIgURE 2: The preparation process of MRF.

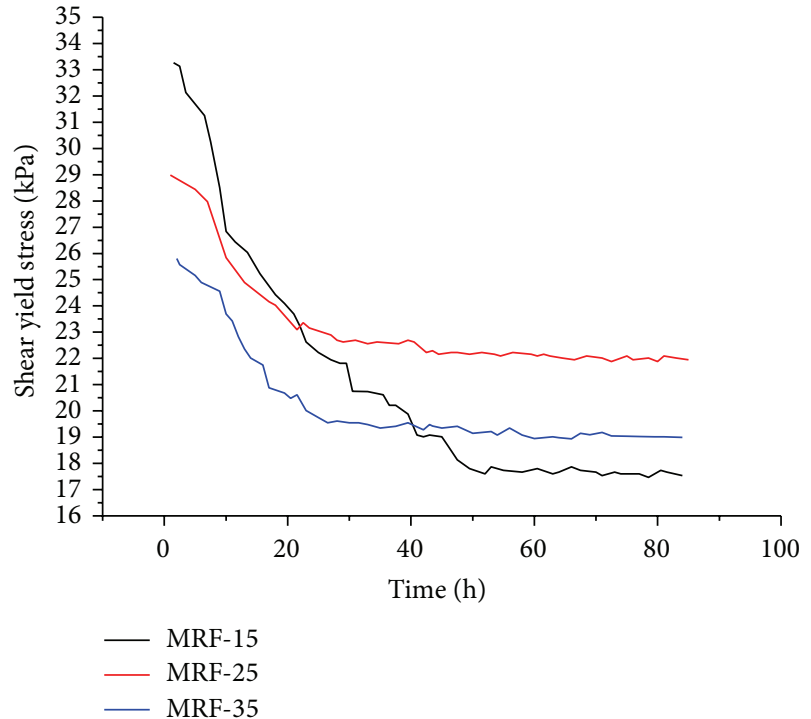

FIGURE 3: Relationship between shear yield stress and working time.

working disks, and $h$ is the spacing of the two disks. The latter part of the formula is the torque produced by $\eta$. Generally, $\eta$ is quite small, and the torque produced by it is below 2 percent of whole transmitted torque of MRF. So, leaving out the latter part, the equation can turn into

$$
T=\frac{2}{3} \pi \tau\left(r_{2}^{3}-r_{1}^{3}\right) .
$$

Thus, according to the maximal transmitted torques $T$ we recorded every one hour during the wear experiments, the corresponding shear yield stresses $\tau$ are obtained. The three MRF samples' change trends of shear yield stresses with working time under the same magnetic field are shown in Figure 3, which clearly show that shear yield stresses of MRF samples decrease with the increasing of working time. But,
TABLE 2: The groups of MRF samples in the wear experiments.

\begin{tabular}{lcc}
\hline Serial number & Control group & Experimental group \\
\hline 1 & MRF-15-a & MRF-15-b \\
2 & MRF-25-a & MRF-25-b \\
3 & MRF-35-a & MRF-35-b \\
\hline
\end{tabular}

according to the curves of three samples, they are leveling off under a given magnetic field. In other words, the MRF will have a nearly constant lower shear yield stress. However, the downward trends of these samples are very different: sample MRF-15, with the highest initial shear yield stress of about $33.5 \mathrm{kPa}$, has the largest decrement, whose final shear yield stress is about $18 \mathrm{kPa}$; as for sample MRF- 25 and sample MRF-35 whose initial values are $29 \mathrm{kPa}$ and $26 \mathrm{kPa}$ while final values are $23 \mathrm{kPa}$ and $19.5 \mathrm{kPa}$, this decrement is smaller.

Finally, we also test the shear stresses of MRF samples obtained before and after the wear experiments under the same exciting currents by using a senior rotary rheometer with magnetorheological fluids module. There are two groups of samples, one of which is a control group including MRF-15a, MRF-25-a, and MRF-35-a obtained before the wear experiments, while the other is experimental group including MRF15-b, MRF-25-b, and MRF-35-b which are obtained after the wear experiments, as shown in Table 2. Besides that, MRF-15b, MRF-25-b, and MRF-35-b correspond, respectively, with MRF-15-a, MRF-25-a, and MRF-35-a. The testing method can be described as follows: keeping a constant shear rate of $10 \mathrm{~s}^{-1}$ while continually increasing the exciting current from $0 \mathrm{~A}$ to $3.5 \mathrm{~A}$, the shear gap is set at $0.5 \mathrm{~mm}$ during the test. The results are illustrated in Figure 4. As we can see from the figure, under the same exciting currents, MRF-15-b, MRF-25-b, and MRF-35-b have lower shear stresses than their counterparts. Moreover, when compared with the samples in control group, the rate of descent in shear stress is the most for MRF-15-b, less for MRF-25-b, and the least for MRF-35-b. 


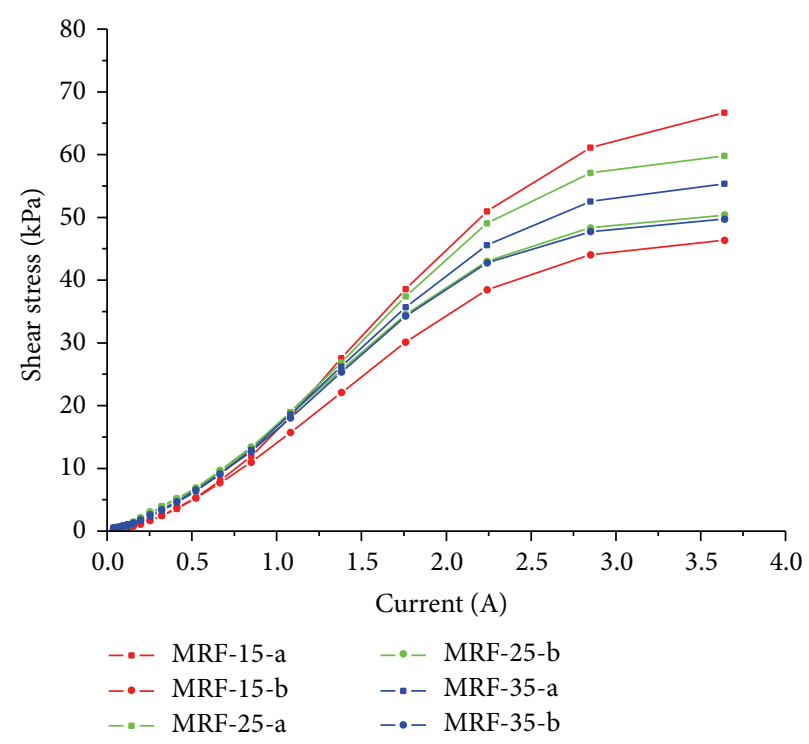

FIGURE 4: Shear stress curves.

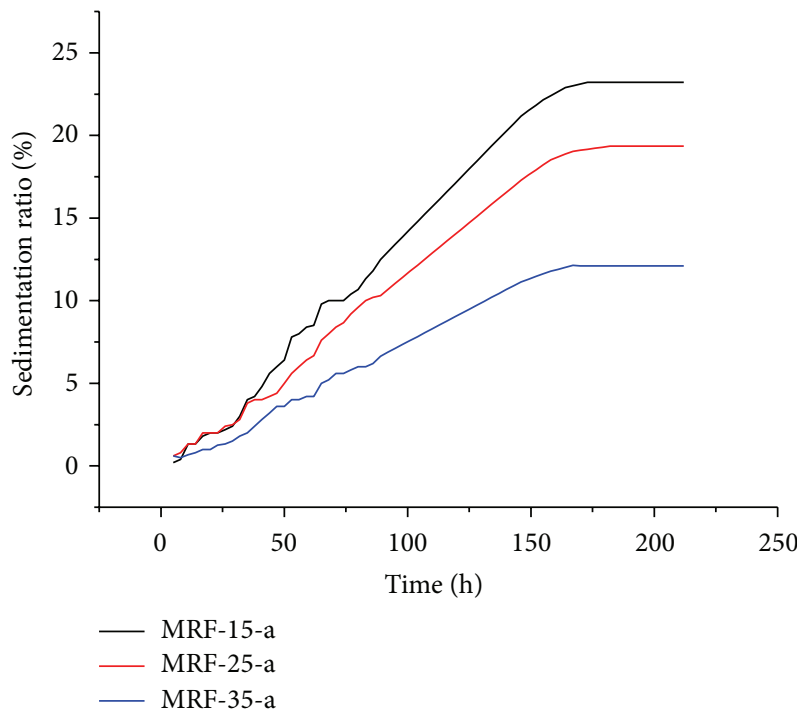

(a) Before the wear experiments

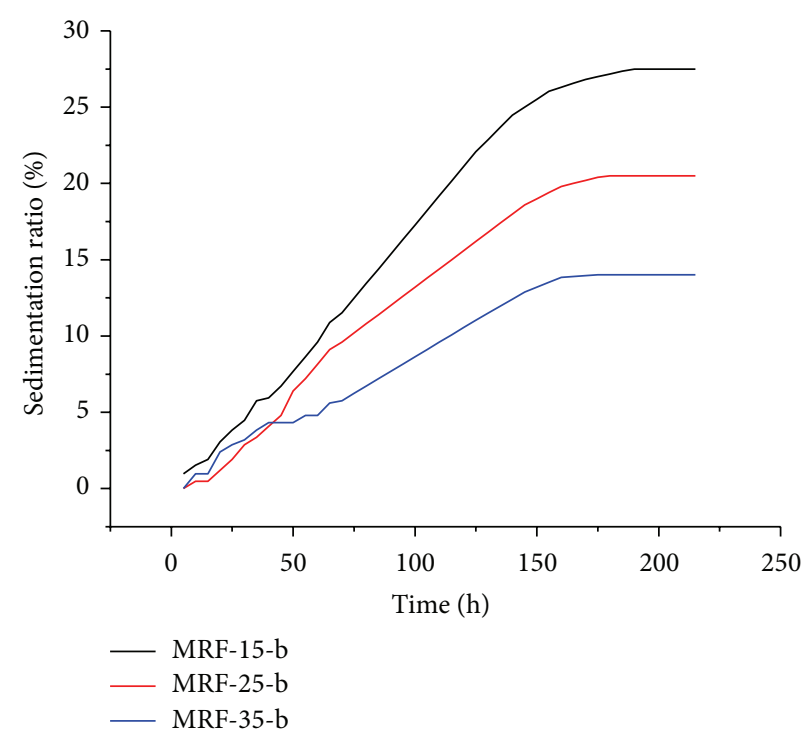

(b) After the wear experiments

FIgURE 5: Sedimentation ratio of MRF.

3.2. Sedimentation Stability of MRF. The sedimentation stability is measured by visual observation method that every MRF sample is put into a $25 \mathrm{~mL}$ measuring cylinder and placed for a period of time (about 10 days). Then, the length of sediment in a certain interval is recorded. The sedimentation stability of MRF can be roughly evaluated by the sedimentation ratio $S$ calculated as follows [22]:

$$
S=\frac{h}{H} \times 100 \%
$$

In this formula, $h$ represents the length of sediment and $H$ represents the total length of MRF.

The sedimentation ratio $S$ of all of the samples is shown in Figure 5. All the sedimentary curves show a similar trend that the sedimentation ratio increases over time and tends to be steady after long settling time. Besides that, they also show that MRF-15-b, MRF-25-b, and MRF-35-b have higher sedimentation ratio compared to MRF-15-a, MRF-25-a, and MRF-35-a. In addition, MRF-35-b has the sedimentation ratio of less than $20 \%$ when it becomes stable. According 


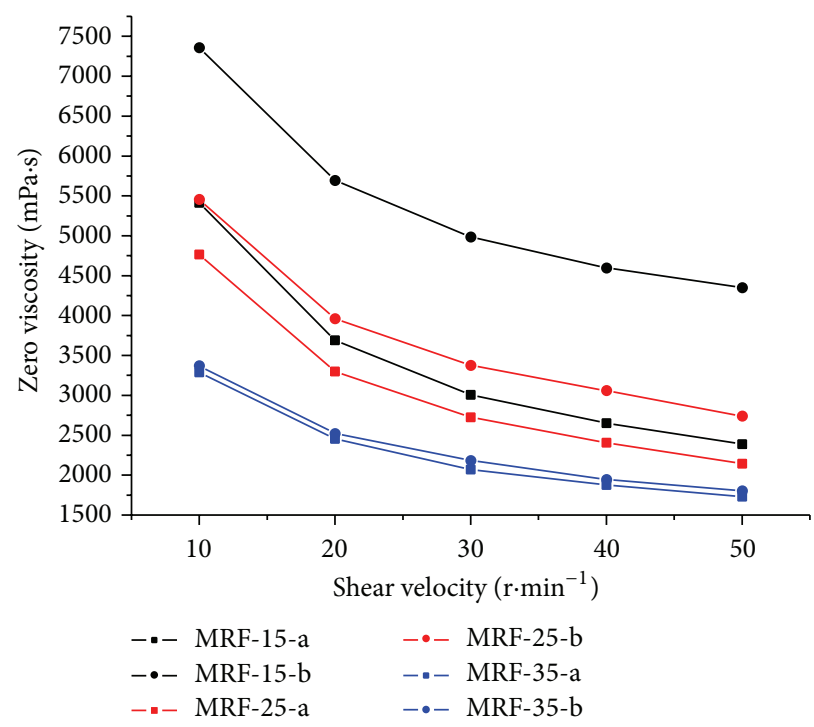

FIGURE 6: Zero-field viscosity of MRF under different shear velocity.

to the research result from Lord Corporation which is specializing in the application of MRF, the well sedimentation ratio of MRF is less than 20\% [23], so we can say that, after the wear experiment, MRF-35-b is doing well in sedimentation stability.

3.3. Zero-Field Viscosity of MRF. Generally, MRF with high performance is requested for lower zero-field viscosity. The values of zero-field viscosity of MRF are measured by SNB1 rotation viscometer. MRF typically acts as free-flowing liquids without the magnetic field and behave similarly to Newtonian fluids [24]. So the zero-field viscosity of MRF is usually related to the shear strain rate; the values of zerofield viscosity for six MRF samples under different shear velocity are shown in Figure 6. It can be seen from the figure that, after the wear experiments, MRF-15-b, MRF-25$b$, and MRF-35-b all have higher viscosity when compared with their counterparts in the control group. Furthermore, under the same wear condition, MRF-15-b and MRF-35-b show, respectively, the maximum and minimum increment of viscosity. So, MRF-35-b is the best one in the experimental group in terms of zero-field viscosity.

3.4. SEM Images of Magnetic Particles. SEM is used to observe the surface morphology of magnetic particles. The morphology of magnetic particles in MRF obtained before the wear experiments is shown in Figure 7(a). Those particles are regular and sphere-like. Figures 7(b) and 7(c) are the SEM images of magnetic particles in MRF that are obtained after the wear experiments. It can be seen that the surface of some particles is worn because of long operating time.

3.5. Discussion. According to the experiment results on the wear property of our prepared MRF, it can be found that the particle size has a significant effect on its wear property. The shear yield stress test results show that MRF-15, MRF-25, and MRF-35 all decrease over time and the decrement gets larger with the increase of particle size. Besides that, a comparison of the shear stresses of MRF samples under the same exciting currents is carried out. The result shows that the shear stress of MRF declines after the wear experiments. Furthermore, by observing the sedimentation ratio and zero-field viscosity of MRF samples, we can find out that they both increased after the experiments. Above all, all the changes get larger with particle size. Lastly, the images taken by SEM indicate that some particles in MRF which have worked for a long time are worn. It can be speculated that the shear stress declines because of those worn particles. Then, the carrier fluids are mixed in the debris of particles. As a consequence of this, the zero-field viscosity increases. What is more, the irregular particles and wear debris can undermine the balance of MRF, thus reducing the stability of sedimentation in some way. In conclusion, big particles are more likely to be damaged although they are very efficient at improving the shear stress of MRF, so MRF with big particles performs less well in the wear property. In this paper, MRF-35 consisting of particles of $1.5-2.8 \mu \mathrm{m}$ in diameter on average is the best selection.

\section{Conclusions and Future Work}

In this paper, several wear experiments were carried out on the test-bed to study the wear property of MRF, and three MRF samples with different size of particles were prepared. Additionally, the effect of particle size on wear property of prepared MRF was studied and discussed to provide references for further study on MRF.

Although the small size particles are good for improving the wear property of MRF, they also decrease the shear stress. So the future work will focus on seeking some antiwear agents which can improve the wear property of MRF to enhance the combination property of it. And finding out the mechanism of the decreasing shear stress caused by wear particles is in the plan. 


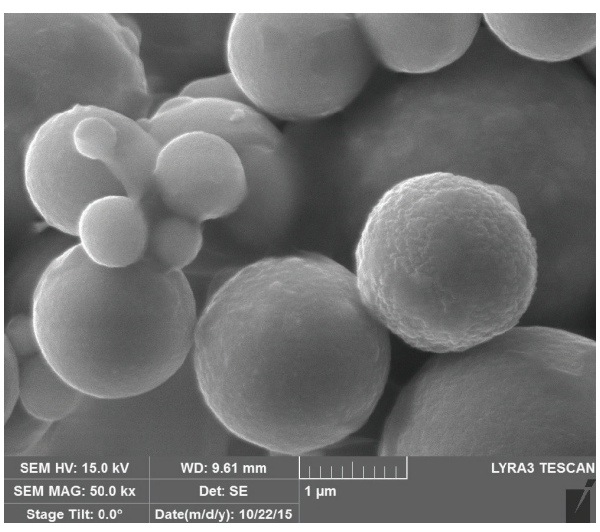

(a)

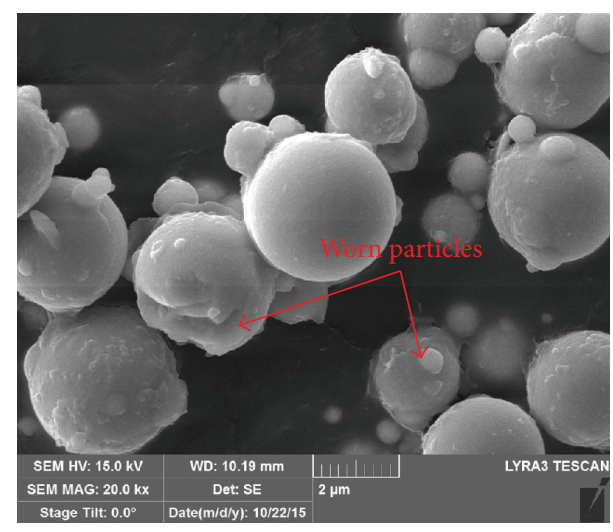

(b)

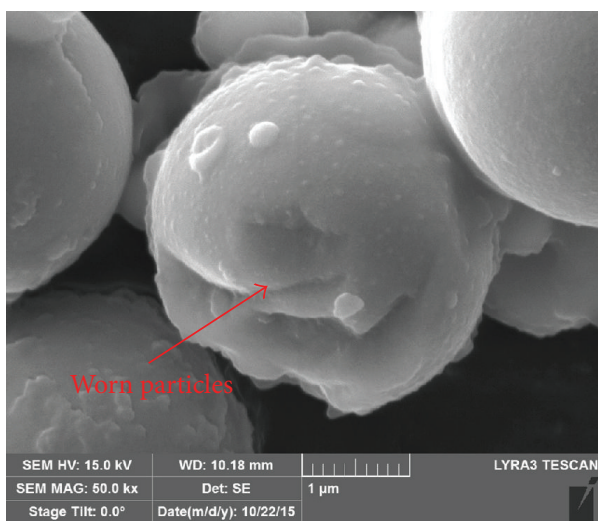

(c)

FIGURE 7: (a) Image of magnetic particles in MRF obtained before wear experiment. (b) Image of worn magnetic particles under 20000 times' amplification. (c) Image of worn magnetic particles under 50000 times' amplification.

\section{Competing Interests}

The authors declare that there is no conflict of interests regarding the publication of this paper.

\section{Acknowledgments}

The support of National Natural Science Foundation of China (no. 51475454), National Natural Science Foundation of Jiangsu Province (no. BK20151144), Open Foundation of the State Key Laboratory of Tribology, Tsinghua University (no. SKLTKF14B06), and Priority Academic Program Development (PAPD) of Jiangsu Higher Education Institutions in carrying out this research is gratefully acknowledged.

\section{References}

[1] X. Chen, X. Zhu, Z. Xu, Y. Lin, and G. He, “The research of the conductive mechanism and properties of magnetorheological fluids," Physica B: Condensed Matter, vol. 418, pp. 32-35, 2013.

[2] A. G. Olabi and A. Grunwald, "Design and application of magneto-rheological fluid," Materials and Design, vol. 28, no. 10, pp. 2658-2664, 2007.

[3] A. L. Kholmetskii, S. A. Vorobyova, A. I. Lesnikovich, V. V. Mushinskii, and N. S. Sobal, "A novel route for the preparation of magnetic fluids," Materials Letters, vol. 59, no. 16, pp. $1993-$ 1996, 2005.

[4] Z. D. Hu, H. Yan, H. Z. Qiu, P. Zhang, and Q. Liu, "Friction and wear of magnetorheological fluid under magnetic field," Wear, vol. 278-279, pp. 48-52, 2012.

[5] G. Dodbiba, H. S. Park, K. Okaya, and T. Fujita, "Investigating magnetorheological properties of a mixture of two types of carbonyl iron powders suspended in an ionic liquid," Journal of Magnetism and Magnetic Materials, vol. 320, no. 7, pp. 1322$1327,2008$.

[6] X. Qiao, J. Zhou, B. P. Binks, X. Gong, and K. Sun, "Magnetorheological behavior of Pickering emulsions stabilized by surface-modified $\mathrm{Fe}_{3} \mathrm{O}_{4}$ nanoparticles," Colloids and Surfaces A: Physicochemical and Engineering Aspects, vol. 412, pp. 20-28, 2012.

[7] L. Bica, "Magnetorheological suspension based on mineral oil, iron and graphite micro-particles," Journal of Magnetism and Magnetic Materials, vol. 283, no. 2-3, pp. 335-343, 2004.

[8] R. Patel, "Mechanism of chain formation in nanofluid based MR fluids," Journal of Magnetism and Magnetic Materials, vol. 323, no. 10, pp. 1360-1363, 2011.

[9] L. E. Udrea, N. J. C. Strachan, V. Bădescu, and O. Rotariu, "An in vitro study of magnetic particle targeting in small blood vessels," Physics in Medicine and Biology, vol. 51, no. 19, pp. 4869-4881, 2006. 
[10] S. N. Madhekar and R. S. Jangid, "Variable dampers for earthquake protection of benchmark highway bridges," Smart Materials and Structures, vol. 18, no. 11, Article ID 115011, 2009.

[11] J.-H. Koo, F. D. Goncalves, and M. Ahmadian, "A comprehensive analysis of the response time of MR dampers," Smart Materials and Structures, vol. 15, no. 2, pp. 351-358, 2006.

[12] A. Milecki and M. Hauke, "Application of magnetorheological fluid in industrial shock absorbers," Mechanical Systems and Signal Processing, vol. 28, pp. 528-541, 2012.

[13] J. Wang and G. Meng, "Experimental study on stability of an MR fluid damper-rotor-journal bearing system," Journal of Sound and Vibration, vol. 262, no. 4, pp. 999-1007, 2003.

[14] X. Liu, D. Wang, H. Lu, and Z. Wang, "Study on macroscopic behavior of silicone oil-based magnetorheological fluids," Optoelectronics and Advanced Materials, Rapid Communications, vol. 7, no. 11-12, pp. 904-909, 2013.

[15] X. Ling and M. Kappl, "Single metal particles nanoscale friction and wear," in Powders and Grains 2009: Proceedings of the 6th International Conference on Micromechanics of Granular Media, vol. 1145, no. 1, pp. 113-116, AIP Publishing, 2009.

[16] J. C. Ulicny, M. P. Balogh, N. M. Potter, and R. A. Waldo, "Magnetorheological fluid durability test-iron analysis," Materials Science and Engineering A, vol. 443, no. 1-2, pp. 16-24, 2007.

[17] J. C. Ulicny, C. A. Hayden, P. M. Hanley, and D. F. Eckel, "Magnetorheological fluid durability test-organics analysis," Materials Science and Engineering A, vol. 464, no. 1-2, pp. 269273, 2007.

[18] H.-T. Pu, F.-J. Jiang, and Z.-L. Yang, "Preparation and properties of soft magnetic particles based on $\mathrm{Fe}_{3} \mathrm{O}_{4}$ and hollow polystyrene microsphere composite," Materials Chemistry and Physics, vol. 100, no. 1, pp. 10-14, 2006.

[19] X. Liu, L. Wang, H. Lu, D. Wang, Q. Chen, and Z. Wang, "A study of the effect of nanometer $\mathrm{Fe}_{3} \mathrm{O}_{4}$ addition on the properties of silicone oil-based magnetorheological fluids," Materials and Manufacturing Processes, vol. 30, no. 2, pp. 204-209, 2015.

[20] X. Liu, Q. Chen, and H. Lu, "Research on the mechanical property of magnetorheological fluids micro-structure under compression working mode," Optoelectronics and Advanced Materials-Rapid Communications, vol. 7, no. 3-4, pp. 231-239, 2013.

[21] X. Liu, H. Lu, Q. Chen, D. Wang, and X. Zhen, "Study on the preparation and properties of silicone oil-based magnetorheological fluids," Materials and Manufacturing Processes, vol. 28, no. 6, pp. 631-636, 2013.

[22] L. Q. Yu, L. J. Zheng, and J. X. Yang, "Study of preparation and properties on magnetization and stability for ferromagnetic fluids," Materials Chemistry and Physics, vol. 66, no. 1, pp. 6-9, 2000.

[23] X. Liu, L. Wang, H. Lu, D. Wang, Q. Chen, and Z. Wang, "Effect of silicone oil viscosity on the properties of magnetorheological fluids," Optoelectronics and Advanced Materials, Rapid Communications, vol. 9, no. 1-2, pp. 226-230, 2015.

[24] L. Wang, X. Liu, Q. Zhang, Y. Ren, and D. Zhang, "A study about the influence of some new additives addition on the properties of magnetorheological fluids," Recent Patents on Materials Science, vol. 8, no. 1, pp. 74-80, 2015. 

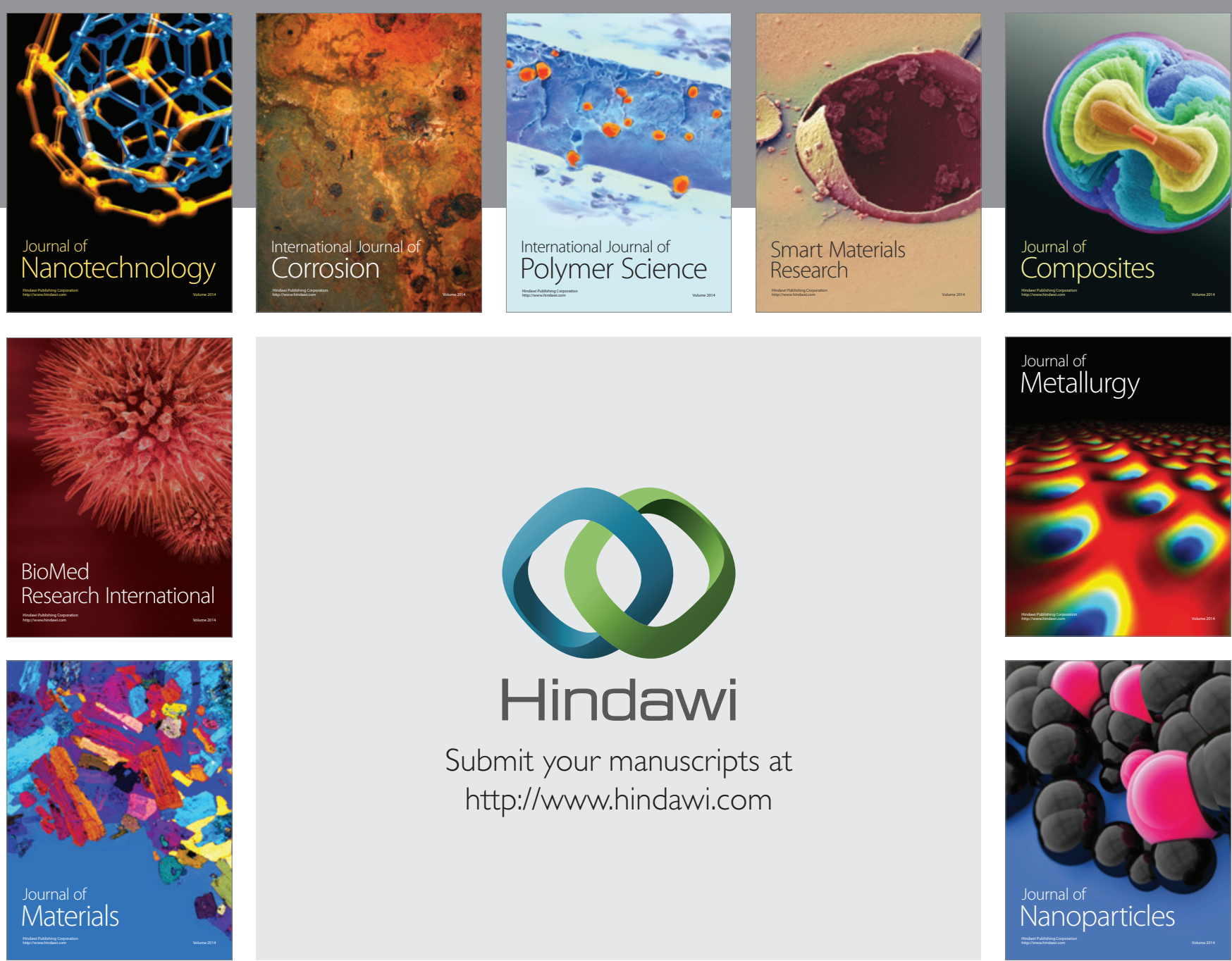

\section{Hindawi}

Submit your manuscripts at

http://www.hindawi.com

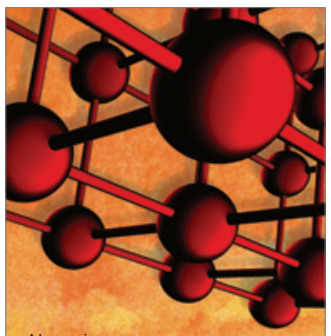

Materials Science and Engineering
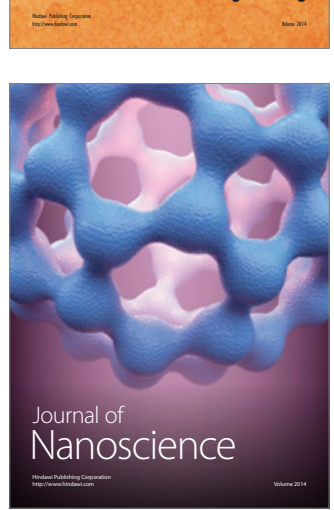
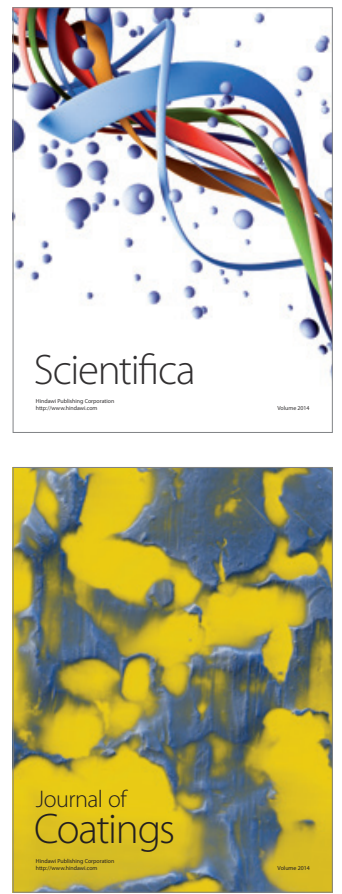
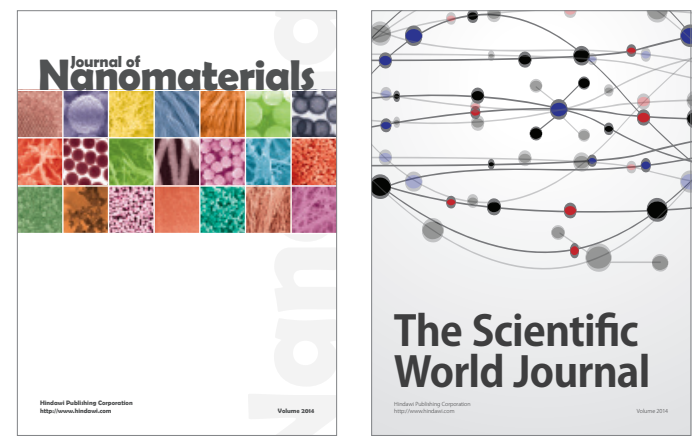

The Scientific World Journal
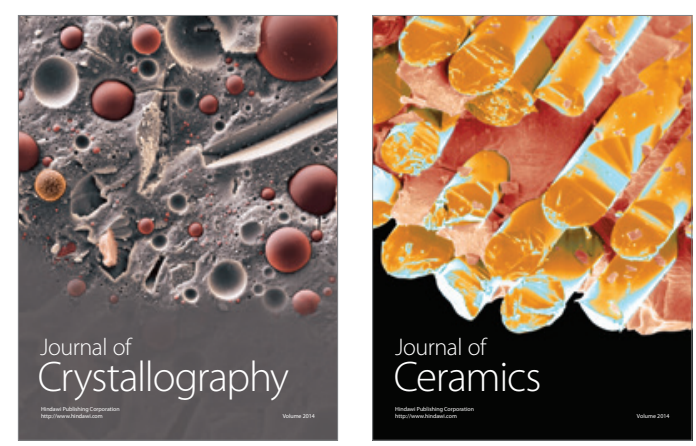
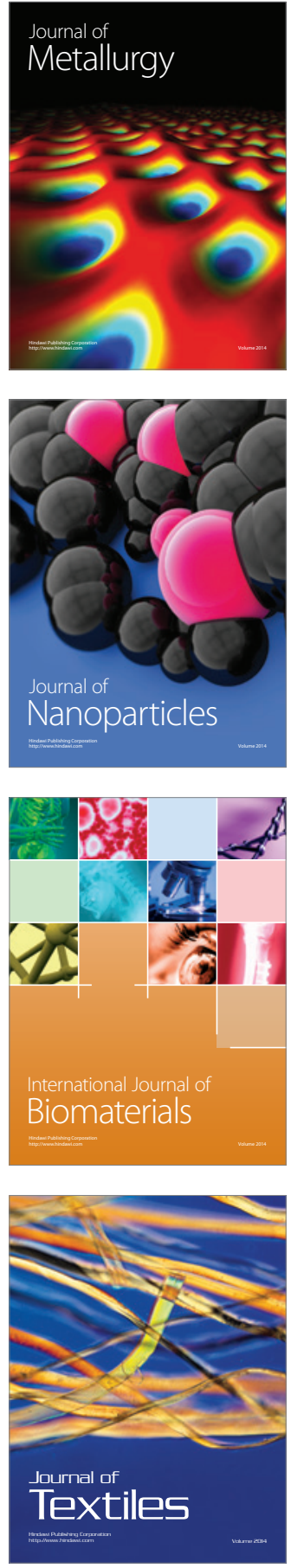